\title{
Spontaneous regression of extruded lumbar disc herniation: Correlation with clinical outcome
}

\author{
Bilge Kesikburun ${ }^{1}$, Emel Eksioglu², Aynur Turan ${ }^{3}$, \\ Emre Adiguzel $^{4}$, Serdar Kesikburun ${ }^{5}$, Aytul Cakci ${ }^{6}$
}

\begin{abstract}
Objective: To evaluate the natural history of lumbar extruded disc with conservative treatment on MRI and to assess relation between the radiologic changes and clinical outcome.

Methods: This prospective observational study was conducted at University of Health Sciences, Diskapi Yildirim Beyazit Training and Research Hospital between May 2015-June 2018. It included consecutive patients who were diagnosed as having lumbar symptomatic extruded disc as shown in MRI. After an average period of $17.0 \pm 7.2$ months, repeat MRI was taken in 40 patients who received only conservative care during follow-up. Changes in the volume of herniated disc was measured. The patients were assigned into 3 groups as follow: (1) non-regression, (2) partial-regression, and (3) complete resolution. Numeric Rating Scale (NRS) pain score, the Oswestry Low Back Pain Disability Index (ODI) and muscle weakness were evaluated.

Results: Based on disc volume of the T2-weighted MR images; four patients (10\%) did not show any regression, six patients $(15 \%)$ had a partial regression, and 30 patients $(75 \%)$ had a complete resolution. Patients with complete resolution showed a significant improvement in the NRS pain score and the ODI score $(p<0,001)$ over time. In patients with partial regression, only the ODI score improved significantly $(p=0,043)$. Non-regression group did not show any improvement in any clinical outcome measure $(p>0,05)$. Changes in the NRS scores over time were significantly higher in complete resolution group compared to non-regression group $(p=0.016)$.

Conclusion: The majority of the patients with extruded lumbar disc herniation might have reduction in size of herniated disc in the long run along with improvement in symptoms and function with conservative care.
\end{abstract}

KEYWORDS: Low back pain, Lumbar disc herniation, Pain, Spine, Spontaneous regression.

How to cite this:

doi: https://doi.org/10.12669/pjms.35.4.346

Kesikburun B, Eksioglu E, Turan A, Adiguzel E, Kesikburun S, Cakci A. Spontaneous regression of extruded lumbar disc herniation: Correlation with clinical outcome. Pak J Med Sci. 2019;35(4):974-980-. doi: https://doi.org/10.12669/pjms.35.4.346

This is an Open Access article distributed under the terms of the Creative Commons Attribution License (http://creativecommons.org/licenses/by/3.0), which permits unrestricted use, distribution, and reproduction in any medium, provided the original work is properly cited.

\section{INTRODUCTION}

Correspondence:

Dr. Bilge Kesikburun, MD,

Department of Physical Medicine and Rehabilitation, Diskapi Yildirim Beyazit Training and Research Hospital, Kazim Karabekir Boulevard, Aslanbey Street, Iskitler, Ankara, Turkey.

Email: drbilgekb@gmail.com

* Received for Publication:

* $\quad 1^{\text {st }}$ Revision Received:

* $\quad 2^{\text {nd }}$ Revision Received:

* Final Revision Accepted:
January 9, 2019

January 14, 2019

April 24, 2019

May 5, 2019
Lumbar disc herniation is a common disorder that causes disability and poor quality of life by limiting functional capacity. Conservative approach or surgical interventions can be used in an individualized treatment plan based on clinical findings and patient preferences. The extruded lumbar disc herniation might generally be considered to be a schedule for surgical treatment, but most of the cases are suggested to be treated successfully by conservative treatment options. ${ }^{1}$ 
The natural history of disc herniation can be spontaneous regression over time as shown on imaging studies. In 1984, Guinto FC Jr et al. ${ }^{2}$ reported the first case of spontaneous regression of lumbar disc herniation. This condition has been described ranging 35\%-100\% for all grades of disc herniation in many studies. ${ }^{3}$ Lumbar disc herniation is enclosed by a granulation tissue comprising newly formed vessels and infiltrating macrophages. It is thought that the neovascularized zone with macrophage infiltration plays major role in the regression. ${ }^{4,5}$ Magnetic resonance imaging (MRI) is suggested as the best option for recognizing disc material and nerve roots. Disc morphology changes over time was shown using MRI in patients with lumbar disc herniation treated conservatively. ${ }^{6-8}$ MRI studies demonstrated that migrated and extruded disc herniation were more reduced in size. ${ }^{9,10}$

The spontaneous regression of herniated disc is a well-known issue. However, the correlation of morphologic changes in herniated disc with clinical outcome is not clear. The aim of this study was to evaluate the natural history of lumbar extruded disc with conservative treatment on MRI and to assess relation between the radiologic changes and clinical outcome.

\section{METHODS}

A prospective observational study was undertaken at University of Health Sciences, Diskapi Yildirim Beyazit Training and Research Hospital between May 2015-June 2018. Patients with low back pain who were recruited from the outpatient clinic were examined to ascertain their eligibility. Sixty four consecutive patients (mean age $50.0 \pm 13.6$ years) who were diagnosed as having lumbar symptomatic extruded disc as shown in MRI were included in the study. That levels with extruded hernia were consistent with symptoms and physical exam findings of the patients. The patients didn't have another discopathy causing symptoms. Extruded disc herniation was defined as 'in at least one plane, any one distance between the edges of the disc material beyond the disc space is greater than the distance between the edges of the base of the disc material'. ${ }^{11}$

Exclusion Criteria: The exclusion criteria were:

1. Previous back surgery,

2. Cauda equina syndrome,

3. Low back pain combined with inflammatory diseases, vertebral fracture, spine infection and tumors
4. Central canal stenosis

5. Spondylolisthesis.

All participants provided written informed consent. The study adhered to the guidelines of the Declaration of Helsinki. The study protocol was approved by the Local Ethics Committee of Hospital.

Demographic data including age, gender, body mass index, duration of low back pain, and comorbidities were recorded. Severity of pain was assessed by numeric rating scale (NRS) ranging from $0 \mathrm{~mm}$ (no pain) to $10 \mathrm{~mm}$ (worst pain). The participants' disability related low back pain was evaluated using a disease-specific functional status questionnaire, Oswestry Low Back Pain Disability Index (ODI). Each question of the ODI is rated on a scale from 0 to 5 points, with a higher score indicating high disability. The ODI scores range from 0 to 50 . The Turkish validity and reliability of ODI was shown. ${ }^{12}$ Muscle weakness was implied as present or absent following a clinical examination at baseline. All patients were re-examined by the same specialist in rehabilitation medicine and reimaged at follow-up visit. During follow-up period, patients received various conservative treatments including medication, physical therapy, exercise, acupuncture, and spinal injection.

MRI: All patients were imaged using a 1.5T MRI scanner (Philips Achieva, Philips Medical Systems, Eindhoven, the Netherlands) with the spine coil in a supine position. Lumbar spinal MRI consisted of sagittal $\mathrm{T} 1 \mathrm{~W}$, sagittal $\mathrm{T} 2 \mathrm{~W}$, and axial $\mathrm{T} 2 \mathrm{~W}$ images. MRI was performed on all patients using the same MRI scanner at baseline and follow-up visit. MRI evaluation was carried out by the same experienced radiologist at baseline and follow-up. The radiologist was blind to the clinical information of the patients at any time point during the study. Volume of herniation $\left(\mathrm{mm}^{3}\right)$ was calculated by measuring the area $\left(\mathrm{mm}^{2}\right)$ in each axial image and multiplying this value by the scan thickness $(4 \mathrm{~mm})$ plus the inter-slice gap $(1 \mathrm{~mm}){ }^{13}$ The patients were assigned into three groups based on change in extruded hernia disc volume as follow:

1. Non-regression: no changes in disc volume at all in sagittal and axial view

2. Partial-regression: markedly decreased herniated disc volume in sagittal and axial view

3. Complete resolution: disappeared herniated disc volume in sagittal and axial view.

Statistical Analysis: Data analysis was performed using SPSS for Mac 20.0 software package program (SPSS Inc., Chicago, IL, United States). Data were 
presented as mean \pm standard deviation [median (interquartile range)] for continuous variables and as frequencies and percentages for categorical variables. Conformity to normal distribution was assessed using the Shapiro-Wilk test. Comparison of the categorical variables was conducted with Chi-Square test. Comparisons within the groups were assessed using the Wilcoxon signed rank test. The Kruskal-Wallis test was used for comparisons between the groups. When statistically significant changes were found between the groups, two-up comparisons were conducted with the Bonferroni corrected Mann Whitney-U test and the results were considered to be statistically significant at $p$ $<0.017$. In all other conditions, a value of $\mathrm{p}<0.05$ was considered statistically significant.

\section{RESULTS}

Sixty four patients were enrolled for the study. Nine patients had lumbar disc surgery and 15 patients had lost to the follow-up visit. A total of 40 patients had a second MRI after the follow-up period, and so included in the statistical analysis (Fig.1). Mean follow-up time was 17.0 \pm 7.2 [14.0(12.0-19.0)] months. There was not significant difference in follow-up times between the groups $(p=0.328)$. Based on disc volume of the T2-weighted MR images; four patients $(10 \%)$ did not show any regression, six patients $(15 \%)$ had a partial regression, and 30 patients $(75 \%)$ had a complete resolution. In partial-regression group, volume of herniated disc reduced from 1433.1 \pm 558.5 [1397,5(855.0-2044.0)] $\mathrm{mm}^{3}$ to $528.5 \pm 331.5[416,0(249.7-920.0)] \mathrm{mm}^{3}$; the percentage of regression was $62.7 \pm 16.4[54,6(50.3-$ $82.3)] \%$. There was no significant difference in baseline demographic and clinical features of the patients between the groups (Table-I). Complete resolution group were inclined to be younger and to have less baseline volume of herniation compared to other groups, however they did not reach a statistically significant importance $(p=0.119$ and $p=0.190$, respectively).

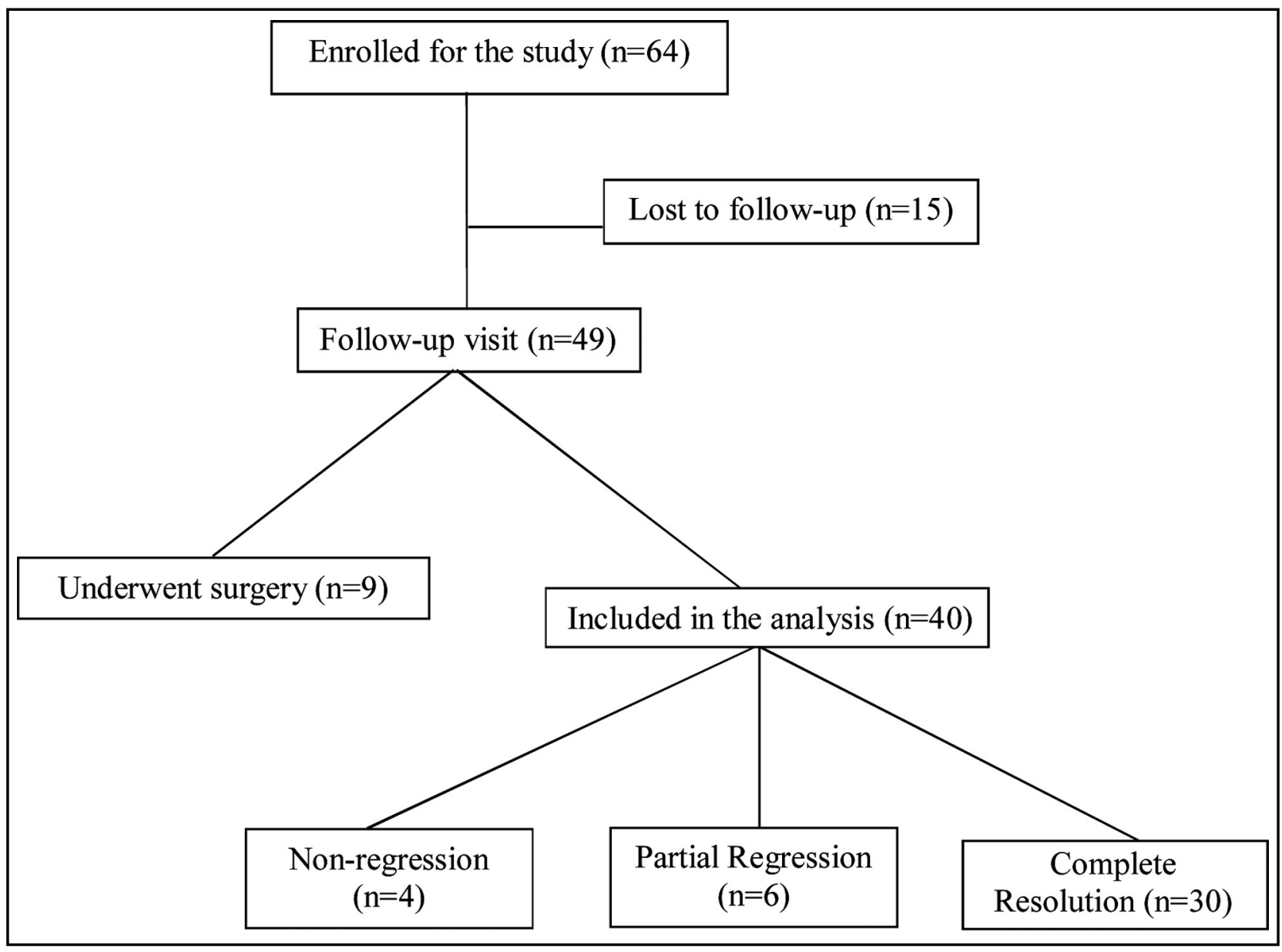

Fig.1: Flow chart. 
Bilge Kesikburun et al.

Table-I: Demographic and clinical features of the patients $(n=40)$.

\begin{tabular}{|c|c|c|c|c|}
\hline & $\begin{array}{c}\text { Non-regression } \\
\quad(n=4)\end{array}$ & $\begin{array}{l}\text { Partial Regression } \\
\qquad(n=6)\end{array}$ & $\begin{array}{c}\text { Complete Resolution } \\
(n=30)\end{array}$ & $p$ \\
\hline Age (years) ${ }^{*}$ & $\begin{array}{c}60.2 \pm 15.5 \\
{[65.5(43.7-71.5)]}\end{array}$ & $\begin{array}{c}54.6 \pm 14.0 \\
{[57.0(39.7-66.7)]}\end{array}$ & $\begin{array}{c}48.3 \pm 10.1 \\
{[50.5(41.7-57.0)]}\end{array}$ & 0.119 \\
\hline Sex & & & & 0.732 \\
\hline Female & $3(75.0 \%)$ & $3(50.0 \%)$ & $12(40.0 \%)$ & \\
\hline Male & $1(25.0 \%)$ & $3(50.0 \%)$ & $18(60.0 \%)$ & \\
\hline $\operatorname{BMI}\left(\mathrm{kg} / \mathrm{m}^{2}\right)^{*}$ & $\begin{array}{c}29.3 \pm 4.2 \\
{[28.8(25.6-33.7)]}\end{array}$ & $\begin{array}{c}27.8 \pm 4.2 \\
{[28.2(23.9-31.9)]}\end{array}$ & $\begin{array}{c}28.7 \pm 5.6 \\
{[27.9(24.9-30.3)]}\end{array}$ & 0.891 \\
\hline Marital Status & & & & 0.386 \\
\hline Married & $4(100.0 \%)$ & $6(100.0 \%)$ & $25(83.3 \%)$ & \\
\hline Single & $0(0.0 \%)$ & $0(0.0 \%)$ & $5(16.7 \%)$ & \\
\hline Occupation & & & & 0.449 \\
\hline No & $4(100.0 \%)$ & $4(66.7 \%)$ & $22(73.3 \%)$ & \\
\hline Yes & $0(0.0 \%)$ & $2(33.3 \%)$ & $8(26.7 \%)$ & \\
\hline $\begin{array}{l}\text { Duration of Pain } \\
\text { (months) }^{*}\end{array}$ & $\begin{array}{c}6.2 \pm 0.9 \\
{[6.5(5.2-7.0)]}\end{array}$ & $\begin{array}{c}6.0 \pm 1.0 \\
{[6.0(5.5-7.0)]}\end{array}$ & $\begin{array}{c}5.9 \pm 1.8 \\
{[6.0(4.7-7.2)]}\end{array}$ & 0.657 \\
\hline \multicolumn{2}{|c|}{ Level of Extruded Disc Herniations } & & & 0.471 \\
\hline L1-L2 & $0(0.0 \%)$ & $0(0.0 \%)$ & $1(3.3 \%)$ & \\
\hline L2-L3 & $0(0.0 \%)$ & $2(33.3 \%)$ & $2(6.7 \%)$ & \\
\hline L3-L4 & $0(0.0 \%)$ & $1(16.7 \%)$ & $2(6.7 \%)$ & \\
\hline L4-L5 & $3(75.0 \%)$ & $2(33.3 \%)$ & $12(40.0 \%)$ & \\
\hline L5-S1 & $1(25.0 \%)$ & $1(16.7 \%)$ & $13(43.3 \%)$ & \\
\hline $\begin{array}{l}\text { Baseline Volume of } \\
\text { Herniation }\left(\mathrm{mm}^{3}\right)^{*}\end{array}$ & $\begin{array}{c}1367.0 \pm 828.8 \\
{[1420.0(567.0-2114.0)]}\end{array}$ & $\begin{array}{c}1433.1 \pm 558.5 \\
{[1397.5(855.0-2044.0)]}\end{array}$ & $\begin{array}{c}1045.6 \pm 736.2 \\
{[844.0(583.0-1327.5)]}\end{array}$ & 0.190 \\
\hline $\begin{array}{l}\text { Follow-up Time } \\
\text { (months) }\end{array}$ & $\begin{array}{c}21.5 \pm 7.5 \\
{[22.0(14.0-28.5)]}\end{array}$ & $\begin{array}{c}18.0 \pm 13.0 \\
{[13.5(10.5-23.7)]}\end{array}$ & $\begin{array}{c}16.2 \pm 6.3 \\
{[13.5(12.0-18.2)]}\end{array}$ & 0.328 \\
\hline
\end{tabular}

BMI: body mass index, ${ }^{*}$ Mean \pm SD [Median (Interquartile Range)].

Table-II: Clinical outcome data.

\begin{tabular}{|c|c|c|c|c|}
\hline & $n$ & Baseline & Follow-up & $p$ \\
\hline \multicolumn{5}{|l|}{ NRS $^{*}$} \\
\hline Non-regression & 4 & $6.0 \pm 1.1[6.0(5.0-7.0)]$ & $4.7 \pm 0.9[6.0(5.0-7.0)]$ & 0.102 \\
\hline Partial Regression & 6 & $6.6 \pm 1.2[6.5(5.7-8.0)]$ & $3,6 \pm 2,2[2,5(2.0-6.2)]$ & 0.063 \\
\hline Complete Resolution & 30 & $6.9 \pm 1.7[7.0(5.7-8.0)]$ & $2.9 \pm 2.0[3.0(1.0-4.2)]$ & $<0.001$ \\
\hline \multicolumn{5}{|l|}{$\mathrm{ODI}^{*}$} \\
\hline Non-regression & 4 & $25.5 \pm 7.5[23.0(20.0-33.5)]$ & $19.2 \pm 7.8[22.0(11.0-24.7)]$ & 0.109 \\
\hline Partial Regression & 6 & $28.6 \pm 10.3[29.5(18.7-38.5)]$ & $14.3 \pm 9.9[12.0(6.2-22.7)]$ & 0.043 \\
\hline Complete Resolution & 30 & $23.3 \pm 11.8[20.0(14.0-32.2)]$ & $12.1 \pm 9.3[11.0(4.0-18.5)]$ & $<0.001$ \\
\hline Muscle Weakness & & & & 0.281 \\
\hline Non-regression & 4 & $0(0.0 \%)$ & $0(0.0 \%)$ & \\
\hline Partial Regression & 6 & $1(16.7 \%)$ & $0(0.0 \%)$ & \\
\hline Complete Resolution & 30 & $5(16.7 \%)$ & $2(6.7 \%)$ & \\
\hline
\end{tabular}

NRS: Numeric Rating Scale, ODI: Oswestry Disability Index, * Mean \pm SD [Median (Interquartile Range)]. 
The comparison of clinical outcome data between baseline and follow-up time points is shown in Table-II. Patients with complete resolution showed a significant improvement in the NRS pain score and the ODI score $(p<0.001)$ over time. In patients with partial regression, only the ODI score improved significantly $(p=0.043)$. Non-regression group did not show any improvement in any clinical outcome measure $(p>0.05)$. Muscle weakness improved in three of five patients in complete resolution group and one patient in partial regression group. Improvement in muscle weakness did not reach a statistically significant change in any group $(p=0.281)$. Changes in the NRS scores over time were significantly higher in complete resolution group compared to non-regression group $(p=0.016)$ (Fig.2). Other two-up comparisons did not show significant differences in the changes in the NRS score and ODI score $(\mathrm{p}>0.05)$.

\section{DISCUSSION}

Current literature suggests lumbar disc herniation may regress spontaneously in a course of time without surgery. The present study inquired whether this chronic painful condition may relieve in the long run with disappearance of the herniated disc material. The findings revealed that three-fourths of the patients with extruded lumbar disc herniation had a complete resolution detected with a second MR imaging after an average follow-up period of 17 months. Those with complete resolution of extruded disc showed a significant pain relief compared to those with nonregressed disc herniation. In addition, low back pain disability improved in patients whose disc herniation regressed completely or partially.

Rate of spontaneous regression in patients with lumbar disc herniation varies depending on the study method, imaging modalities, inclusion criteria, follow-up time, morphologic classification of disc herniations and regression grading of herniations. Hong SJ et al..$^{14}$ investigated the resorption rate of massive lumbar disc herniation in patients treated with transforaminal epidural steroid injection and reported that $85.7 \%(24 / 28)$ of patients had a partial or complete reduction in size of the herniation. Ahn et $a .^{6}$ reported that that $67 \%$ of patients with extruded disc herniation had dramatic resolution after a mean of 4.3 months follow-up time. Benson et al. ${ }^{15}$ revealed that $83 \%(29 / 35)$ of the patients with massive lumbar disc herniation had a complete and sustained recovery at an average follow-up time of 23.2 months. Bush $\mathrm{K}$ et al. ${ }^{8}$ found that 64 of the 84 herniated/sequestered intervertebral discs $(76.1 \%)$ showed a partial or complete resolution at one year on follow-up CT scanning. Komori Het al. ${ }^{16}$ reported that the patients with extruded disc herniation had $30.7 \%(8 / 26)$ partial regression and no complete resolution after a mean of 6.3 months follow-up time. Takada E et al. ${ }^{17}$ showed all 17 patients with extruded disc herniation $(100 \%)$ had regression more than half in size with a two-year follow-up. It may be suggested that higher rate of regression and resolution for extruded lumbar disc herniation may be showed with a longer follow-up time. Chiu CC et al. ${ }^{3}$ reported that extruded and sequestrated type herniation were predictive factors for spontaneous regression of lumbar disc herniation and showed that the rate of extruded and sequestrated discs were 7.8 times higher than bulging or protruded discs. The present study included only the patients

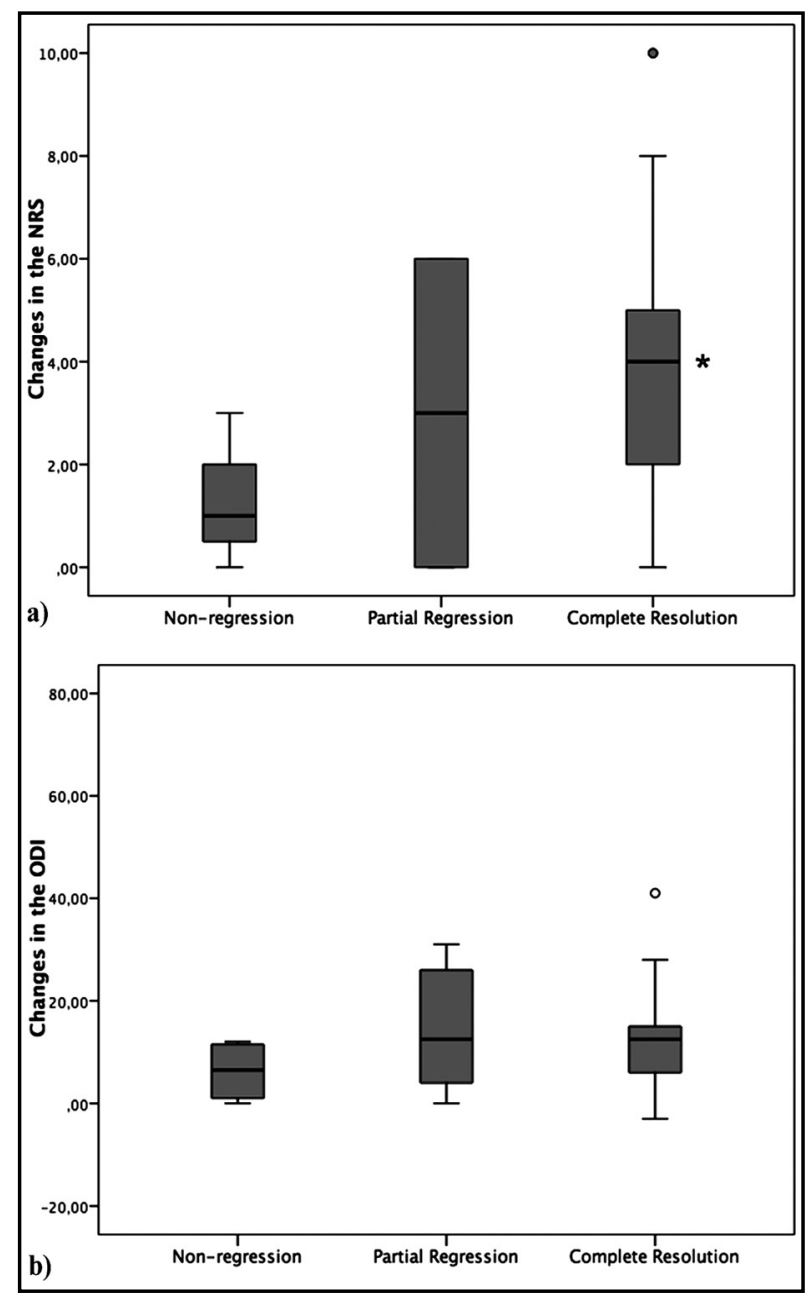

Fig.2: Comparison of changes in the NRS (a) and ODI (b) scores over time between the groups.

* Significant difference in complete resolution group compared to nonregression group ( $\mathrm{p}<0.017$, Mann-Whitney test). 
with extruded disc herniation. In addition, this study had relatively a longer follow-up time. So, the rate of spontaneous regression, especially complete resolution, might be found higher compared to the previous studies.

The relation between disc regression and clinical improvement has not been accounted for well. It was explained that not only compression of a nerve root by a herniated disc is responsible for the symptoms, but also the release of inflammatory cytokines and the composition of the herniated disc may contribute to the pain. ${ }^{18}$ Seo JY et al. ${ }^{19}$ reported that volumetric changes in herniated discs were not significantly correlated with clinical outcomes, while Hong SJ et al. ${ }^{14}$ showed that patients had significantly symptomatic improvements with disc regression. The present study found that pain and disability improved significantly with disc resorption.

Extruded disc herniation is generally an alerted condition for both clinicians and patients. Thus, surgery might be sometimes scheduled early due to pain without presence of absolute surgery indications such as progressive motor deficit and cauda equine. However, the present findings suggest that conservative management of the cases may lead to regression of extruded disc together with improved clinical outcome in the long run. Considering the likelihood of postoperative complications such as recurrence and failed back surgery syndrome, ${ }^{20,21}$ conservative care might be prolongable for the patients whom refused early surgical treatment option, and meanwhile the patients might be monitored for a possible surgery decision with careful consideration of absolute indications. A recent prospective cohort study comparing surgical and conservative treatment for lumbar disc herniation suggested faster pain relief with surgery, but similar benefit with both treatments in the mid-term and long-term follow up..$^{22}$ Atlas SJ et al. ${ }^{23}$ in their long-term lumbar spine study recommended individualized treatment plan required patients with lumbar disc herniation and their physicians to integrate clinical findings with patient preferences based on their symptoms and goals.

Limitations of the study: The present study has achieved the goals that it aimed to substantiate. On the other hand, there are some limitations of this study. First, only the patients who chose not to undergo surgery were included in the study, introducing a potential bias in the population studied. Second, there was not a standardized time for second imaging, but it was longer than a year almost for everyone and it might be considered enough for showing long-term results. Third, even it was an observational cohort study, the rate of patients lost to follow-up is higher than expected. On the other hand, 40 patients were still enough to highlight the results of the study. Finally, patients underwent various conservative treatments during the follow-up period. So, the present results failed to show the impact of a specific conservative technique. Further prospective studies focused on a single conservative treatment with a control group might be performed to illuminate this issue.

\section{CONCLUSION}

The majority of the patients with extruded lumbar disc herniation might have reduction in size of herniated disc along with an improvement in symptoms and function with conservative care. So prolonged conservative treatment might be taken into consideration for the patients who do not prefer early surgery in the absence of absolute neurogenic complications secondary to nerve compression.

Declaration of interests: The authors declared no conflicts of interest with respect to the authorship and/or publication of this article.

Funding: None.

\section{REFERENCES}

1. Saal JA, Saal JS. Nonoperative treatment of herniated lumbar intervertebral disc with radiculopathy: An outcome study. Spine (Phila Pa 1976). 1989;14(4):431-437.

2. Guinto FC Jr, Hashim H, Stumer M. CT demonstration of disk regression after conservative therapy. Am J Neuroradiol. 1984;5(5):632-633.

3. Chiu CC, Chuang TY, Chang $\mathrm{KH}, \mathrm{Wu} \mathrm{CH}$, Lin $\mathrm{PW}$, Hsu WY. The probability of spontaneous regression of lumbar herniated disc: a systematic review. Clin Rehabil. 2015;29(2):184-195. doi: 10.1177/0269215514540919.

4. Haro H, Shinomiya K, Komori H, Okawa A, Saito I, Miyasaka N, et al. Upregulated expression of chemokines in herniated nucleus pulposus resorption. Spine (Phila Pa 1976). 1996;21(14):647-652.

5. Kato T, Haro H, Komori $H$, Shinomiya K. Sequential dynamics of inflammatory cytokine, angiogenesis inducing factor and matrix degrading enzymes during spontaneous resorption of the herniated disc. J Orthop Res. 2004;22(4):895900. doi: 10.1016/j.orthres.2003.11.008.

6. Ahn SH, Park HW, Byun WM, Ahn MW, Bae JH, Jang $\mathrm{SH}$, et al. Comparison of clinical outcomes and natural morphologic changes between sequestered and large central extruded disc herniations. Yonsei Med J. 2002;43(3):283-290. doi: 10.3349/ymj.2002.43.3.283. 
7. Bozzao A, Gallucci M, Masciocchi C, Aprile I, Barile A, Passariello R. Lumbar disk herniation: MR imaging assessment of natural history in patients treated without surgery. Radiology. 1992;185(1):135-141. doi: 10.1148/ radiology.185.1.1523297.

8. Bush K, Cowan N, Katz DE, Gishen P. The natural history of sciatica associated with disc pathology: a prospective study with clinical and independent radiologic followup. Spine (Phila Pa 1976). 1992;17(10):1205-1212. doi: 10.1080/1355297X.1993.11719717.

9. Ahn SH, Ahn MW, Byun WM. Effect of the transligamentous extension of lumbar disc herniations on their regression and the clinical outcome of sciatica. Spine (Phila Pa 1976). 2000;25(4):475-480.

10. Komori H, Shinomiya K, Nakai O, Yamaura I, Takeda S, Furuya K. The natural history of herniated nucleus pulposus with radiculopathy. Spine (Phila Pa 1976). 1996;21(2):225-229.

11. Fardon DF, Williams AL, Dohring EJ, Murtagh FR, Gabriel Rothman SL, Sze GK. Lumbar disc nomenclature: version 2.0: recommendations of the combined task forces of the North American Spine Society, the American Society of Spine Radiology, and the American Society of Neuroradiology. Spine (Phila Pa 1976). 2014;39(24):1448-1465. doi: 10.1097/BRS.0b013e3182a8866d.

12. Yakut E, Duger T, Oksuz C, Yorukan S, Ureten K, Turan $\mathrm{D}$, et al. Validation of the Turkish version of the Oswestry Disability Index for patients with low back pain. Spine (Phila Pa 1976). 2004;29(5):581-585.

13. Autio RA, Karppinen J, Niinimaki J, Ojala R, Kurunlahti M, Haapea $M$, et al. Determinants of spontaneous resorption of intervertebral disc herniations. Spine (Phila Pa 1976). 2006;31(11):1247-1252. doi: 10.1097/01. brs.0000217681.83524.4a.

14. Hong SJ, Kim DY, Kim H, Kim S, Shin KM, Kang SS. Resorption of Massive Lumbar Disc Herniation on MRI Treated with Epidural Steroid Injection: A Retrospective Study of 28 Cases. Pain Physician. 2016;19(6):381-388.

15. Benson RT, Tavares SP, Robertson SC, Sharp R, Marshall RW. Conservatively treated massive prolapsed discs: A 7-year follow-up. Ann R Coll Surg Engl. 2010;92(2):147-153. doi: 10.1308/003588410X12518836438840.

16. Komori H, Okawa A, Haro H, Muneta T, Yamamoto H, Shinomiya K. Contrast-enhanced magnetic resonance imaging in conservative management of lumbar disc herniation. Spine (Phila Pa 1976). 1998;23(1):67-73.

17. Takada E, Takahashi M, Shimada K. Natural history of lumbar disc hernia with radicular leg pain: Spontaneous MRI changes of the herniated mass and correlation with clinical outcome. J Orthop Surg (Hong Kong). 2001;9(1):1-7. doi: $10.1177 / 230949900100900102$.
18. Omarker K, Myers RR. Pathogenesis of sciatic pain: role of herniated nucleus pulposus and deformation of spinal nerve root and dorsal root ganglion. Pain. 1998;78(2):99-105. doi: 10.1016/S0304-3959(98)00119-5.

19. Seo JY, Roh YH, Kim YH, Ha KY. Three-dimensional analysis of volumetric changes in herniated discs of the lumbar spine: does spontaneous resorption of herniated discs always occur? Eur Spine J. 2016;25(5):1393-1402. doi: 10.1007/s00586-014-3587-1.

20. Baber $Z$, Erdek MA. Failed back surgery syndrome: current perspectives. J Pain Res. 2016;9:979-987. doi: 10.2147/JPR. S92776.

21. McGirt MJ, Ambrossi GL, Datoo G, Sciubba DM, Witham $\mathrm{TF}$, Wolinsky JP, et al. Recurrent disc herniation and longterm back pain after primary lumbar discectomy: review of outcomes reported for limited versus aggressive disc removal. Neurosurgery. 2009;64(2):338-344. doi: 10.1227/01. NEU.0000337574.58662.E2.

22. Gugliotta M, da Costa BR, Dabis E, Theiler R, Jüni $P$, Reichenbach $S$, et al. Surgical versus conservative treatment for lumbar disc herniation: a prospective cohort study. BMJ Open. 2016;6(12):e012938. doi: 10.1136/ bmjopen-2016-012938

23. Atlas SJ, Keller RB, Wu YA, Deyo RA, Singer DE. Long-term outcomes of surgical and nonsurgical management of sciatica secondary to a lumbar disc herniation: 10 years results from the main lumbar spine study. Spine (Phila Pa 1976). 2005;30(8):927-935. doi: 10.1097/01.brs.0000158954.68522.2a.

\section{Author's Contribution:}

KB: Data collection and manuscript writing.

EE: Design the study.

TA: Analysis of data.

KS: Statistical analysis and critical review.

AE: Data collection.

CA: Critical review and final approval.

Authors:

1. Bilge Kesikburun, MD,

2. Emel Eksioglu, MD,

Associate Professor,

3. Aynur Turan, MD,

Associate Professor, Department of Radiology,

4. Emre Adiguzel, MD,

Associate Professor,

5. Serdar Kesikburun, MD,

Associate Professor,

Aytul Cakci, MD,

1,2,6: University of Health Sciences,

Dıșkapı Yıldırım Beyazıt Training and Research Hospital, Department of Physical Medicine and Rehabilitation, Ankara, Turkey

3: $\quad$ University of Health Sciences, Dıșkapı Yıldırım Beyazıt Training and Research Hospital, Department of Radiology, Ankara, Turkey

4,5: University of Health Sciences, Gulhane School of Medicine, Department of Physical Medicine and Rehabilitation, Gaziler Physical Therapy and Rehabilitation Training and Research Hospital, Ankara, Turkey. 